\title{
Recent progress of the sign problem in the Onishi formula
}

\author{
Noritaka Shimizu ${ }^{1, \star}$, Takahiro Mizusaki ${ }^{2}$, and Makito $\mathrm{Oi}^{2}$ \\ ${ }^{1}$ Center for Nuclear Study, The University of Tokyo, 7-3-1 Hongo, Bunkyo-ku, Tokyo 113-0033, Japan \\ ${ }^{2}$ Institute of Natural Sciences, Senshu University, 3-8-1 Kanda-Jinbocho, Chiyoda-ku,Tokyo 101-8425, \\ Japan
}

\begin{abstract}
The Hartree-Fock-Bogoliubov (HFB) method is one of the major methods to study a nuclear wave function. We discuss the sign problem in the Onishi formula, which emerges in calculating the overlap between two HFB wave functions. The origin of this problem is elucidated by expanding the overlap as a polynomial function. This article is based on Ref.[1].
\end{abstract}

\section{Introduction}

The Hartree-Fock-Bogoliubov (HFB) wave function is one of the most efficient and essential forms to describe a quantum many-body wave function which has strong pairing correlations. While the HFB wave function is usually defined as a quasi-particle vacuum, in the present work we begin with the following form of the HFB wave function:

$$
\left|\phi^{(k)}\right\rangle=e^{\sum_{i<j} Z_{i j}^{(k)} c_{i}^{\dagger} c_{j}^{\dagger}}|-\rangle=e^{\hat{Z}^{(k)}}|-\rangle, \quad \hat{Z}^{(k)}=\sum_{i<j} Z_{i j}^{(k)} c_{i}^{\dagger} c_{j}^{\dagger},
$$

where $c_{i}^{\dagger}$ and $|-\rangle$ are the creation operator of the single-particle state $i$ and the vacuum, respectively. This wave function is parametrized by a skew-symmetric matrix $Z^{(k)}$.

The overlap of two HFB wave functions is obtained by the Onishi formula [2] as

$$
\left\langle\phi^{(0)} \mid \phi^{(1)}\right\rangle=\sqrt{\operatorname{Det}\left(I-Z^{(0) *} Z^{(1)}\right)},
$$

where $I$ is the unit matrix. However, since a square root appears in Eq.(2), the sign of this equation is not determined. Hereafter we call this sign uncertainty "sign problem" in the Onishi formula. This problem can be serious when we perform angular-momentum projection.

\section{Remedies for the sign problem}

A lot of effort had been paid to solve the sign problem till now (e.g. [3]). Among them, in 1980's, two remedies were suggested. One is called "continuity method" [4]. In this method, the continuity of the overlap kernel as a function of the Euler angles is utilized to determine the sign. This procedure,

^e-mail: shimizu@cns.s.u-tokyo.ac.jp 
however, becomes very complicated, especially in high-spin states. The other method was suggested by Neergård and Wüst. This method is based on the fact that the matrix $Z^{0 *} Z^{1}$ has pair-wisely degenerate eigenvalues [5]. In this method, however, since this matrix has no particular symmetry such as hermiticity, its diagonalization might cause numerical instability. Thus, these two methods are useful in numerical calculations, while neither of them is a perfect solution.

In 2009, Robledo proposed a sophisticated formula utilizing Pfaffian [6] as

$$
\left\langle\phi^{(0)} \mid \phi^{(1)}\right\rangle=(-1)^{N(N+1) / 2} \operatorname{Pf}\left(\begin{array}{cc}
Z^{(1)} & -I \\
I & -Z^{(0) *}
\end{array}\right),
$$

where $N$ is the number of the single-particle states, namely the dimension of $Z^{(k)}$. In Eq.(3), the overlap is provided by an N/2-th order polynomial of the matrix elements of $Z^{(1)}$ and $Z^{(0) *}$. Thus, this formula apparently does not have the sign problem and is simple without numerical instability.

By comparing Eqs.(2) and (3), one question can arise: why does the transcendental function (square root) appear in Eq.(2) while it can be represented in a polynomial function in Eq. (3)? Since the square root is also the origin of the sign problem, it is worth discussing the derivation of the Onishi formula to elucidate how the square root appears in the derivation.

\section{Onishi formula and polynomial expansion}

While the derivation of the Onishi formula is not shown in Ref.[2], we show the derivation utilizing the linked cluster theorem [7] in this section. Further details of the derivation can be found in Ref.[1].

According to the linked cluster theorem, the overlap is provided by the exponential function of the summation of the connected terms. Some lengthy calculations lead to the connected term given as

$$
\ln \left(\left\langle\phi^{0} \mid \phi^{1}\right\rangle\right)=\left\langle\phi^{0} \mid \phi^{1}\right\rangle_{c}=\sum_{k=1}^{\infty}-\frac{1}{2 k} \operatorname{Tr}\left(Y^{k}\right)
$$

where $Y=Z^{(0) *} Z^{(1)}$ and the subscript $c$ denotes the connected term. Note that the summation up to the infinite number of terms appears in Eq.(4) although only finite number of terms appear in Eq.(3). The Onishi formula is obtained by the linked-cluster theorem as

$$
\left\langle\phi^{0} \mid \phi^{1}\right\rangle=\exp \left(\left\langle\phi^{0} \mid \phi^{1}\right\rangle_{c}\right)=\exp \left(-\frac{1}{2} \sum_{k=1}^{\infty} \frac{1}{k} \operatorname{Tr}\left(Y^{k}\right)\right)=\exp \left(\frac{1}{2} \operatorname{Tr} \ln (I-Y)\right)=\sqrt{\operatorname{Det}(I-Y)} .
$$

The rightmost term is nothing but the Onishi formula. The coefficient $\frac{1}{2}$ in the middle of Eq.(5) brings about the square root of the Onishi formula. In the Neergård and Wüst method, the pairwise degeneracy of $Y$ cancels this coefficient.

On the other hand, the overlap is obtained by expanding the exponential function as

$$
\left\langle\phi^{0} \mid \phi^{1}\right\rangle=\left\langle-\left|\exp \left(\hat{Z}^{(0) \dagger}\right) \exp \left(\hat{Z}^{(1)}\right)\right|-\right\rangle=\sum_{k=0}^{N / 2} \frac{1}{(k !)^{2}}\left\langle-\left|\left(\hat{Z}^{(0) \dagger}\right)^{k}\left(\hat{Z}^{(1)}\right)^{k}\right|-\right\rangle
$$

where the Pauli exclusion principle terminates the summation concerning $k$ at the $N / 2$-th order. The $(N / 2+1)$-th and the higher-order terms become zero thanks to the Pauli exclusion principle. It is consistent with Robledo's formula. More explicitly, each term of this polynomial function is shown in Fig.1. 


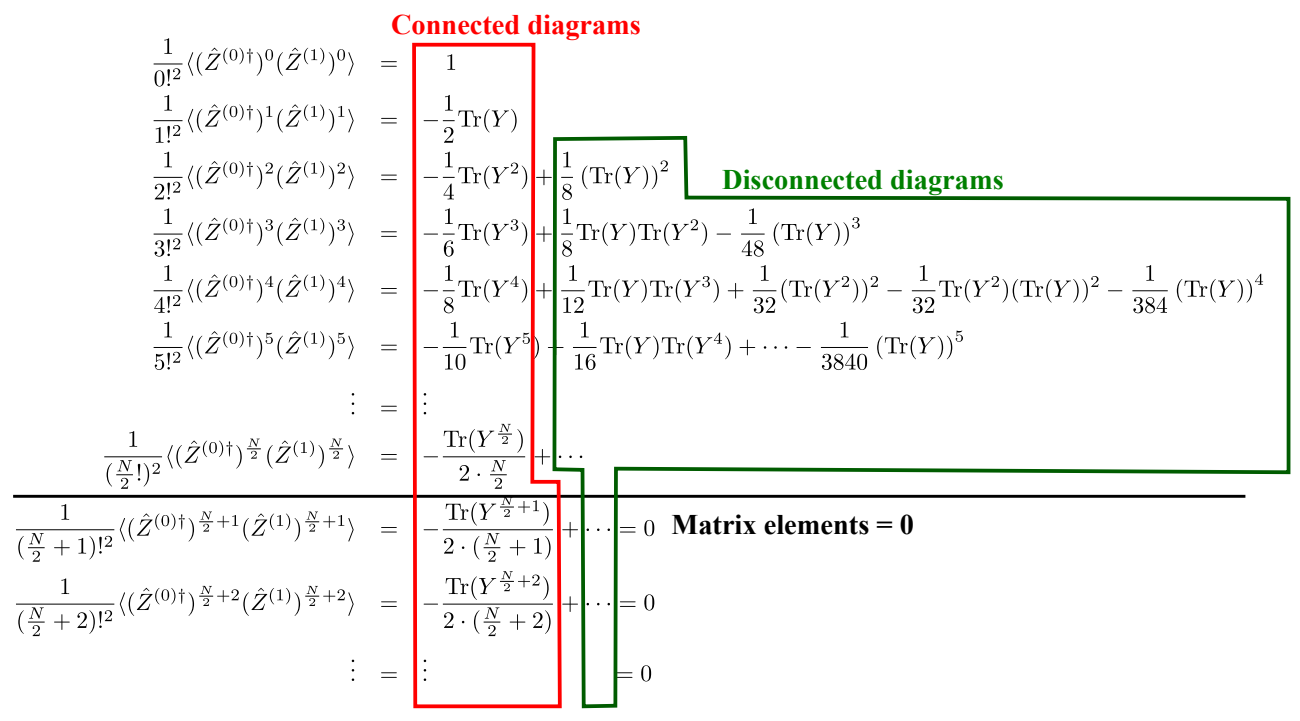

Figure 1. Polynomial expansion of the overlap of two HFB wave functions.

In each term, the corresponding connected term is enclosed by the red line. Even though the value of the higher terms in the left-hand-side equation is zero, the connected term is finite. In the Onishi formula, the connected terms enclosed by the red line is summed up infinitely. This causes the transcendental function, although the higher-order term becomes zero due to the cancellation of the connected term and the disconnected terms.

\section{Summary}

By comparing the Onishi formula to the polynomial expansion of the overlap of two HFB wave functions explicitly, we clarified that the sign problem is caused by the apparent infinite summation of the polynomial terms, which can be reduced to the finite summation in Robledo's formula. This consideration brought about a new idea of the efficient way of the computation of the HFB overlap, which will be published in near future [8].

NS acknowledges KAKENHI Grant 17K05433 and the priority issue 9 to be tackled by using Post-K computer.

\section{References}

[1] T. Mizusaki, M. Oi, and N. Shimizu, Phys. Lett. B 779, 237 (2018).

[2] N. Onishi and S. Yoshida, Nucl. Phys. 80, 367 (1966).

[3] B. Bally and T. Duguet, Phys. Rev. C 97, 024304 (2018).

[4] K. Hara, Y. Hayashi, and P. Ring, Nucl. Phys. A 358, 14 (1982).

[5] K. Neergård and E. Wüst, Nucl. Phys. A 402, 311, (1983).

[6] L.M. Robledo, Phys. Rev. C 79, 021302(R) (2009).

[7] C. Bloch, Nucl. Phys. 7, 451 (1958).

[8] M. Oi, T. Mizusaki, N. Shimizu, and Y. Sun, in preparation. 